This item was submitted to Loughborough's Research Repository by the author.

Items in Figshare are protected by copyright, with all rights reserved, unless otherwise indicated.

\title{
Chemical functionalization of graphene oxide for improving mechanical and thermal properties of polyurethane composites
}

PLEASE CITE THE PUBLISHED VERSION

http://dx.doi.org/10.1016/j.matdes.2015.07.101

\section{PUBLISHER}

(C) Elsevier

VERSION

SMUR (Submitted Manuscript Under Review)

\section{PUBLISHER STATEMENT}

This work is made available according to the conditions of the Creative Commons Attribution-NonCommercialNoDerivatives 4.0 International (CC BY-NC-ND 4.0) licence. Full details of this licence are available at: https://creativecommons.org/licenses/by-nc-nd/4.0/

\section{LICENCE}

CC BY-NC-ND 4.0

\section{REPOSITORY RECORD}

Jing, Qifei, Wanshuang Liu, Yongzheng Pan, Vadim V. Silberschmidt, Lin Li, and ZhiLi Dong. 2019. "Chemical Functionalization of Graphene Oxide for Improving Mechanical and Thermal Properties of Polyurethane Composites". figshare. https://hdl.handle.net/2134/19458. 
Chemical functionalization of graphene oxide for improving mechanical and thermal properties of polyurethane composites

Qifei Jing, ${ }^{\mathrm{a}, \mathrm{b}, \mathrm{c}}$, Wanshuang Liu ${ }^{\mathrm{a}}$, Vadim V. Silberschmidt ${ }^{\mathrm{c}}$, Lin $\mathrm{Li}^{\mathrm{d}, *}$, ZhiLi Dong $^{\mathrm{ab},{ }^{*}}$

${ }^{\mathrm{a}}$ School of Materials Science and Engineering, Nanyang Technological University, 50 Nanyang Avenue, Singapore 639798, Singapore

${ }^{\mathrm{b}}$ Institute for Sports Research, Nanyang Technological University, 50 Nanyang Avenue, Singapore 639798, Singapore

${ }^{\mathrm{c}}$ Wolfson School of Mechanical and Manufacturing Engineering, Loughborough University, Loughborough, Leicestershire LE11 3TU, UK

${ }^{\mathrm{d}}$ School of Mechanical and Aerospace Engineering, Nanyang Technological University, 50 Nanyang Avenue, Singapore 639798, Singapore

* Corresponding authors. Tel.: +65 67906727

E-mail address: zldong@ntu.edu.sg (Z. Dong)

Address: N4.1-01-04, School of Materials Science and Engineering, Nanyang Technological University, 50 Nanyang Avenue, Singapore 639798, Singapore 


\begin{abstract}
Graphene oxide (GO) was chemically functionalized to manufacture polyurethane (PU) composites with improved mechanical and thermal properties. In order to achieve a well exfoliated and stable GO suspension in organic solvent, 4, 4'- methylenebis(phenyl isocyanate) and polycaprolactone diol - two monomers used to synthesize polyurethane were employed to functionalize GO sequentially. The obtained functionalized GO (FGO) could form homogeneous dispersions in DMF solvent and the PU matrix, as well as provide a good compatibility with the latter. The most efficient improvement in mechanical properties was achieved when $0.4 \mathrm{wt} \%$ FGO was added into the PU matrix, corresponding to increases in the tensile stress, elongation at break and toughness by $34.2 \%, 27.6 \%$ and $64.5 \%$, respectively (compared with those of PU). Regarding the thermal stability, FGO/PU 1 wt\% showed the largest enhancement, with $\mathrm{T}_{2 \%}$ and $\mathrm{T}_{50 \%} 16{ }^{\circ} \mathrm{C}$ and $21{ }^{\circ} \mathrm{C}$ higher than those for PU, respectively. A significant improvement in both mechanical properties and thermal stability of FGO/PU composites should be attributed to homogeneous dispersion of FGO in the PU matrix and strong interfacial interaction between them.
\end{abstract}

Keywords: Composites; Dispersion; Graphene oxide; Polyurethane.

\title{
1. Introduction
}

Graphene - a one-atom-thick hexagonal lattice of $\mathrm{sp}^{2}$ carbon atoms - exhibits a twodimensional sheet-like structure. Research into graphene has been one of the fastest growing areas due to its many fascinating properties such as ultrahigh thermal and electrical conductivity, excellent thermal stability, high specific surface area and remarkable mechanical strength. [1, 2] Graphene was proven theoretically [3] and experimentally [4] to be the strongest material developed so far. Significant efforts have been put into exploring possibilities to use graphene as nanofiller to develop multi-functional polymer composite materials for various applications, such as electromagnetic interference-shielding materials, shape-memory devices, materials for drug release, actuators and infrared-triggered sensors. [5-10] To obtain defect-free, high-quality graphene sheets, the most widely used approaches are chemical vapour deposition [11, 12] and micromechanical cleavage of graphite [13]. Still, these approaches show a low production yield and are time consuming. In this situation, 
graphene oxide (GO), originated from the exfoliation of graphite oxide, has been studied in many researches as an alternative to graphene. The established advantages of GO in production yield and cost [14] make it an attractive candidate as a nanofiller used in polymer composites.

GO, having the same framework as graphene, contains massive oxygen functional groups such as epoxide, hydroxyl, carboxyl and carbonyl groups located at its edges and basal planes [15]. Hence, GO can be readily dispersed in water and form a stable colloidal suspension due to its strong hydrophilicity $[16,17]$. However, it could benefit only fabrication of GO-based/ aqueous polymer composites. The process of exfoliation of GO in organic solvents is not favoured, presumably due to strong interlayer hydrogen bonds originated from those attached oxygen functional groups. Strong interactions between the adjacent GO layers could prevent penetration of organic molecules into the interlayer spaces, thus preventing full exfoliation of GO in organic solvents. Ruoff et al. [18] consider the hydrophilicity of GO as an obstacle to prepare high-performance GO-reinforced polymer composites in organic solvents. In their work, reactions between organic isocyanates, and hydroxyl and carboxyl groups were taken advantage of to reduce the amount of hydrogen bonds with donor groups on GO sheets, thus weakening the strength of interlayer hydrogen bonding and hydrophilicity of GO. As a result, the isocyanate-treated GO can be fully exfoliated in some organic solvents, e.g., dimethylformamide (DMF), after a mild ultrasonication.

Thermoplastic polyurethane (PU) is an important class of polymers, which has been widely used in various applications such as foams, coatings, elastomers and adhesives. PU has a block copolymer structure, with polyol as soft segment and isocyanate and chain extender as hard segments. A molecular structure of PU could be easily adjusted to fulfil different property requirements [19]. It is commonly acknowledged that two factors should be mainly concerned in order to prepare high-performance nanofiller/polymer composites: (1) dispersion of nanofillers in the polymer matrix; (2) interfacial interactions between nanofillers and the matrix. The nanofillers tend to aggregate due to strong van der Waals forces acting between them, hindering their homogeneous dispersion in a polymer matrix. Besides, a lack of strong interfacial interactions between the nanofillers and matrix would greatly compromise the reinforcement effect of the nanofillers. One feasible and effective strategy to promote the reinforcement of composites is through chemical functionalization of nanofillers [20-22]. Among chemical functionalization methods, grafting molecules and/or polymer chains onto the surface of graphene sheets was studied for many polymers [23-25]. 
The grafted molecules and/or polymer chains can usually enhance dispersion of graphene sheets in the polymer matrix and interfacial interactions between them.

In this work, GO/PU composites were prepared via a solution mixing method in DMF. In order to obtain a homogeneous dispersion of GO in DMF solvent, as well as a strong interfacial interaction between GO and PU matrix, 4, 4'- methylenebis(phenyl isocyanate) (MDI) and polycaprolactone diol (PCL) molecules were selectively used to functionalize GO sequentially. On the one hand, MDI and PCL functionalization of GO was expected to improve dispersion of GO in DMF solvent, which could consequently improve the dispersion of GO in the PU matrix. On the other hand, since MDI and PCL were the monomers of synthesizing PU, a good compatibility between the functionalized GO (FGO) and the PU matrix was also expected, considering their structural similarity. Detailed characterizations of FGO were conducted and presented to confirm the successful functionalization of MDI and PCL on GO surface. In addition, mechanical properties and thermal stability were tested to demonstrate the reinforcement effect of FGO on the composite materials.

\section{Experimental}

\subsection{Materials}

Graphene oxide (purity > 99\%) was provided by XFNANO Materials Tech Co., Ltd. (Nanjing, China); it was synthesized from graphite powders using a common Hummers method. PCL (average $\mathrm{Mn} \sim 530 \mathrm{~g} / \mathrm{mol}$ and $\sim 2000 \mathrm{~g} / \mathrm{mol}$ ), MDI (molecular weight 250.25 g/mol), 1,4-butanediol (BD, molecular weight $90.12 \mathrm{~g} / \mathrm{mol}$ ), and dibutyltin dilaurate (DBTDL) were all obtained from Sigma-Aldrich. DMF (Tritech Scientific), anhydrous DMF (Tritech Scientific) and anhydrous acetone (Tritech Scientific) were used as received.

\subsection{Functionalization of $G O$}

Before using, GO was vacuum freeze-dried to reduce the amount of stored water in its $\pi-$ stacked structure [26]. In a typical operation, $300 \mathrm{mg}$ GO was added into a round-bottom flask followed by $150 \mathrm{ml}$ anhydrous DMF under a nitrogen atmosphere to form a homogeneous suspension with the aid of an ultrasonication bath. Then, a $20 \mathrm{ml}$ anhydrous DMF solution of MDI (5 g, $20 \mathrm{mmol}$ ) was mixed with the obtained suspension. The mixture was subsequently heated to $80^{\circ} \mathrm{C}$ and kept for $24 \mathrm{~h}$ with magnetic stirring under nitrogen. 
After that, the mixture was poured into massive anhydrous acetone to coagulate a wet solid. The solid was filtered, washed with additional anhydrous acetone and anhydrous DMF sequentially. Then, the resulting solid was immediately re-dispersed in $150 \mathrm{ml}$ anhydrous DMF with the assistance of ultrasonication. Subsequently, a $20 \mathrm{ml}$ anhydrous DMF solution of PCL (5 g, $9.4 \mathrm{mmol}$ ) was added and stirred for $36 \mathrm{~h}$ at $80{ }^{\circ} \mathrm{C}$ under nitrogen. After $36 \mathrm{~h}$ the reaction mixture was coagulated in massive anhydrous acetone to get a solid product. The product was further filtered, washed repeatedly with DMF to obtain FGO. The obtained FGO was then dispersed in DMF by ultrasonication to form a stable stock suspension with a concentration of $0.71 \mathrm{mg} / \mathrm{ml}$ for a later use. The schematic of FGO preparation is presented in Fig. 1.

\subsection{Preparation of FGO/PU composites}

In this work, the prepolymerization method [27] was adopted to synthesize thermoplastic PU. MDI and PCL were used as the two monomers, with DBTDL and BD employed as the catalyst and chain extender, respectively. The feed molar ratio of MDI: PCL: BD was 6: 1: 5, corresponding to a hard-segment content of $49.4 \mathrm{wt} \%$. The solution mixing method was used to prepare a series of FGO/PU composites with different FGO contents - $0.1 \mathrm{wt} \%, 0.4 \mathrm{wt} \%$, $0.7 \mathrm{wt} \%$ and $1 \mathrm{wt} \%$. First, a certain volume of the as-prepared FGO/DMF stock suspension was added in a flask and then the synthesized PU ( $\sim \mathrm{g})$ was added into the suspension, based on the required FGO content in the composite. Considering different volumes of solvent in the series of FGO/DMF suspensions, additional DMF was supplied to ensure the same volume of solvent for each sample. After PU was completely dissolved with the assistance of magnetic stirring, a homogenizer was used at $9000 \mathrm{r} / \mathrm{min}$ for $15 \mathrm{~min}$, followed by a bath ultrasonication for $40 \mathrm{~min}$, to ensure good homogeneity of the suspensions. Finally, the FGO/PU composite films were obtained through solution casting. The content of FGO in the composite was calculated based on the initial FGO amount in the stock suspension. As a control group, GO/PU composite (0.4 wt\%) was prepared according to the same procedure.

\subsection{Characterization}

FTIR spectroscopic measurements used the FTIR Frontier (Perkin Elmer) system equipped with Attenuated Total Reflection (ATR). For sample preparation, the powder sample was first mixed and ground with potassium bromide. Then, the mixture was pressed into a round 
transparent pellet in a pellet-forming die. The functionalization degree of FGO and thermal stability of the composite films were determined by thermo-gravimetric analysis (TGA) tests (TGA Q500). The powder samples were first kept at $100^{\circ} \mathrm{C}$ for $10 \mathrm{~min}$ to remove the absorbed water and then were heated again to $600^{\circ} \mathrm{C}$ at a speed of $10^{\circ} \mathrm{C} / \mathrm{min}$ under nitrogen; the films samples were heated with the latter regime. Powder X-ray diffraction (XRD) tests were conducted using a Shimadzu X-ray diffractometer $(\mathrm{Cu} \mathrm{K \alpha})$ with step scanning $\left(0.02^{\circ}\right.$, $0.6 \mathrm{~s}$ dwell time, $40 \mathrm{kV}$ ) over a $2 \theta$ range of $5-35^{\circ}$. Field-Emission Scanning Electron Microscopy (FESEM) (JSM-7600F) was used to observe morphological features of GO and FGO. Before observations, the powder samples were sputtered with a thin layer of platinum using a sputter coater. Transmission Electron Microscopy (TEM) studies were conducted with a Carl Zeiss LIBRA ${ }^{\circledR} 120$ in-column energy filter TEM system equipped with an integrated OMEGA filter. Leica Ultracut UCT was used to microtome the film samples in order to obtain flakes with a thickness of about 50-100 nm for TEM analysis. Tensile Tester Instron 5567 was utilized to conduct the tensile tests. The films samples for these tests were cut according to ASTM D638, corresponding to a gauge length of $9.5 \mathrm{~mm}$. The tests were conducted at room temperature, and five specimens were tested for each composite film.

\section{Results and Discussion}

\subsection{Characterization of FGO and GO}

The FTIR tests were conducted to confirm the successful functionalization of GO, as shown in Fig. 2. When organic isocyanates were used to functionalize GO, amides [28] and carbamate esters [29] would be formed derivatized with the carboxyl and hydroxyl groups, respectively. The typical features of GO were observed in the spectra. The peaks at $3431 \mathrm{~cm}^{-1}$, $1727 \mathrm{~cm}^{-1}$, and $1060 \mathrm{~cm}^{-1}$ were associated with stretching ofO-H, C=O and C-O-C, respectively. After treatment with MDI and PCL sequentially, many new peaks appeared for FGO, compared with GO. The $\mathrm{C}=\mathrm{O}$ stretching vibration of $\mathrm{GO}$ at $1727 \mathrm{~cm}^{-1}$ shifted towards a lower wavenumber and was divided into two small peaks at $1698 \mathrm{~cm}^{-1}$ and $1670 \mathrm{~cm}^{-1}$, corresponding to $\mathrm{C}=\mathrm{O}$ stretching of the carbamate esters and amides, respectively. Together with the new peaks at $1598 \mathrm{~cm}^{-1}$ and $1310 \mathrm{~cm}^{-1}$, which were due to $\mathrm{N}-\mathrm{H}$ bending and C-N stretching, the reaction between the isocyanates and GO was confirmed [30]. Besides, the peaks at $2927 \mathrm{~cm}^{-1}$ and $2864 \mathrm{~cm}^{-1}$ were attributed to asymmetrical and symmetrical stretching vibrations of $-\mathrm{CH}_{2^{-}}$[31], and the peak at $757 \mathrm{~cm}^{-1}$ was attributed to the $-\mathrm{CH}_{2}$ - bending 
vibration (4 or more $-\mathrm{CH}_{2}^{-}$) [30]. It is noteworthy that the peaks for isocyanate group ($\mathrm{N}=\mathrm{C}=\mathrm{O}$ ), ranging from $2275-2263 \mathrm{~cm}^{-1}$ [18], were not found in FGO, indicating that the $\mathrm{N}=\mathrm{C}=\mathrm{O}$ groups of MDI reacted completely with the hydroxyl and carboxyl groups. The FTIR results confirmed that both MDI and PCL were covalently functionalized to the surface of GO.

The functionalization degree of FGO was determined with the TGA tests. Figure 3 exhibits the TGA curves of GO, FGO and PU. Prior to heating, the samples were held isothermal at $100^{\circ} \mathrm{C}$ for $10 \mathrm{~min}$ to remove the absorbed water [32]. It can be observed in Fig. 3 that GO was not thermally stable. Its major mass loss appeared in the temperature range from $150^{\circ} \mathrm{C}$ to $300^{\circ} \mathrm{C}$, associated with pyrolysis of the massive labile oxygen functional groups on its surface [33]. In contrast, the total mass loss of FGO could be divided into two major stages. The first stage should be due to pyrolysis of the residual oxygen functional groups from GO. It is clearly seen that the amount of oxygen functional groups in FGO was significantly decreased, presumably due to the following two reasons: first, the isocyanate groups from MDI would react with the hydroxyl and carboxyl groups on the surface of GO, as confirmed by FTIR tests, thus reducing GO to a certain extent; second, the solvothermal reduction [34, 35] could also play a role in decreasing the amount of oxygen functional groups since GO experienced a relative high temperature and long time in the DMF solvent during the functionalization process. The second stage exhibited a similar weight-loss temperature range to that of the PU film, indicating that the mass loss at that stage was attributed to thermal decomposition of covalently grafted MDI and PCL on the surface of GO. Estimated from the TGA curves, FGO contained approximately $40 \mathrm{wt} \%$ of covalently functionalized MDI and PCL.

The XRD tests were performed to observe a structural change between FGO and GO; Fig 4 presents XRD patterns of GO (a) and FGO (b) powders. A typical diffraction peak of GO was observed at approximately $2 \theta=10.8^{\circ}$, corresponding to d-spacing of $0.82 \mathrm{~nm}$. However, no characteristic intense diffraction peak was observed for FGO, indicating that the covalently functionalization of GO by MDI and PCL could effectively prevent them from restacking. It is worth mentioning that the broad peaks appeared at about $21^{\circ}$ for both FGO and GO, originated from the glass substrate used as sample holder in XRD tests.

The FESEM images show the morphological features of GO and FGO. As can be seen in Fig. 5, GO sheets exhibited wrinkled structures and smooth surfaces, while FGO sheets presented comparatively rough surfaces and were covered by addends due to the covalently grafted MDI and PCL, as confirmed by the FTIR and TGA results. 


\subsection{Dispersions in solvent and PU matrix}

Figure 6 presents digital photographs of GO and FGO dispersed in DMF (0.5 mg/ml) for 5 min and 14 days after ultrasonication. As can be observed in Fig. 6a, both GO and FGO formed homogeneous colloidal suspensions in DMF after the ultrasonication process. However, after 14 days, a visible precipitate could be observed in the vial with GO in the DMF solvent, whereas the suspension of FGO in it remained stable and showed no visible precipitate. Apparently, FGO exhibited a better dispersion ability in the DMF solvent than GO [18]. The massive oxygen functional groups on the surface of GO sheets could form strong interlayer hydrogen bonds, which did not favour penetration of organic molecules of the solvent into the interlayer spaces. After chemical functionalization of GO with MDI and PCL, the amount of available hydrogen-bond donor groups decreased significantly, thus weakening the interlayer interactions in FGO, which contributed to the exfoliation of FGO in the DMF solvent. Meanwhile, the covalently grafted MDI and PCL on the surface of the FGO sheets could effectively prevent the graphene backbones from direct contact [33], which was also beneficial to the exfoliation of FGO in the solvent.

Generally, performance of a composite relies greatly on a dispersion state of nanofillers in the polymer matrix. As shown in Fig. 7, dispersion of GO and FGO in the PU matrix was observed with TEM. In the GO/PU (0.4 wt\% nanofillers) composite, the GO sheets could be clearly observed, indicating their stacked structure, which was consistent with the XRD results. In contrast, in the FGO/PU composite with the same fraction of nano-reinforcement, the FGO sheets appeared obscure indicating their good exfoliation and fine dispersion in the matrix. From the TEM images, the better exfoliation and dispersion of FGO in the PU matrix than those of GO was confirmed. As shown above, FGO possessed a better dispersion ability in the DMF solvent than GO. Considering that the fabrication of the composites was processed in this solvent, the dispersion of FGO in the PU matrix should benefit from its good dispersion in it. Moreover, the covalently grafted MDI and PCL on the surface of FGO could improve compatibility between FGO and the PU matrix, contributing to better dispersion of FGO in the PU matrix.

\subsection{Mechanical properties}


Typical stress-strain curves obtained in mechanical tests for PU, as well as the FGO/PU and GO/PU composites with different fraction of nanofillers are presented in Fig. 8. Since all the composite films exhibited a nonlinear elastic behaviour, the tangent modulus was calculated by taking the slope of the curve at low strains (0-8\%), while the toughness was determined by calculating the area under the curve. Figures 9 and 10 provide detailed information about the main mechanical parameters - tensile strength, modulus, elongation at break and toughness. In general, the introduction of FGO into the PU matrix could significantly improve its mechanical properties. It can be seen that the most efficient improvement was achieved for the FGO/PU $0.4 \mathrm{wt} \%$ composite. Its tensile stress, elongation at break and toughness were 19.6 MPa, 1035.3\% and 129.6 MPa, respectively, corresponding to increases by $34.2 \%, 27.6 \%$ and $64.5 \%$ compared with those of PU. Further addition of FGO into the PU matrix led to a decrease of these values, which was presumably due to the aggregation of FGO. Besides, the modulus of the FGO/PU composite increased gradually with the increasing FGO content, reaching a peak of 29.6 MPa at $1 \mathrm{wt} \%$. The significant improvement of mechanical properties in the FGO/PU composites was attributed to good dispersions of FGO in the PU matrix, as well as the strong interfacial interactions between nanofiller and the polymer. The covalently grafted MDI and PCL on the surface of FGO could form interfacial hydrogen bonding with the PU matrix, resulting in an effective load transfer from the matrix to FGO under external stress. Moreover, as can be seen in Fig. 10, FGO provided a higher extent of improvements than GO for the same loading content (0.4 wt\%) overall. The toughness and elongation at break of FGO/PU with 0.4 wt\% nanofillers (129.6 MPa and 1035.3\%) were 19.6\% and 21.8\% higher than those of GO/PU with the same fraction of reinforcement (108.4 MPa and 850.3\%), respectively. Interestingly, the initial modulus of this FGO/PU composite was smaller than that of the GO/PU compsoite, which might be due to covalently grafted MDI and PCL on the surface of FGO. Compared with GO, the FGO sheets covered with small molecules and soft chains could act as a "plasticizer" in the matrix, resulting in a lower stiffness yet higher toughness and ductility of the composite.

\subsection{Thermal stability}

Thermal stability of the studied FGO/PU composites was characterized with TGA tests; the results are shown in Fig. 11. It can be clearly seen that the curves shifted towards higher temperatures after adding FGO into the PU matrices. To assess the thermal stability, the temperatures corresponding to $2 \%\left(\mathrm{~T}_{2 \%}\right)$ and $50 \%\left(\mathrm{~T}_{50 \%}\right)$ weight loss of the composites were 
taken as the criteria [36]. The largest improvement of thermal stability was achieved for the FGO/PU composite with $1 \mathrm{wt} \%$ FGO. The $\mathrm{T}_{2 \%}$ and $\mathrm{T}_{50 \%}$ for it $\left(255^{\circ} \mathrm{C}\right.$ and $271^{\circ} \mathrm{C}$ ) increased by $16^{\circ} \mathrm{C}$ and $21^{\circ} \mathrm{C}$, respectively, compared with those for $\mathrm{PU}\left(327{ }^{\circ} \mathrm{C}\right.$ and $348{ }^{\circ} \mathrm{C}$ ). Apparently, the addition of FGO to the PU matrices could greatly reinforce the thermal stability of the composites, owing to the so-called "tortuous-path" effect of graphene sheets, which delayed the escape of volatile degradation products and char formation [37].

\section{Conclusions}

In this work, GO was functionalized with the two monomers of PU: MDI and PCL, sequentially. The successful functionalization was confirmed with the FTIR, TGA, XRD and FESEM measurements. After the functionalization, the amount of oxygen functional groups on the surface of GO was reduced. The covalently grafted MDI and PCL on the surface of FGO helped not only to improve dispersion of FGO in the DMF solvent and the PU matrix, but also to provide a good compatibility between FGO and the PU matrix. The most efficient improvement in mechanical properties was achieved when $0.4 \mathrm{wt} \%$ FGO was added into the PU matrix, resulting in the increases in tensile stress, elongation at break and toughness of 34.2\%, 27.6\% and 64.5\% compared with those of PU. Regarding the thermal stability, the FGO/PU composite with $1 \mathrm{wt} \%$ nanofillers showed the largest reinforcement with $\mathrm{T}_{2 \%}$ and $\mathrm{T}_{50 \%} 16^{\circ} \mathrm{C}$ and $21^{\circ} \mathrm{C}$ higher than those of $\mathrm{PU}$, respectively. A proper chemical functionalization of graphene sheets is crucial for their exfoliation and homogeneous dispersion in the organic solvent and polymer matrix, as well as a strong interfacial interaction between the graphene sheets and the polymer matrix. The method using monomer-functionalized graphene oxide to prepare graphene-based polymer composite with enhanced performance is very promising.

\section{Acknowledgements}

The financial support from Institute for Sports Research of Nanyang Technological University (NTU) is appreciated. The XRD, FESEM and TEM work were performed at the Facility for Analysis, Characterization, Testing and Simulation (FACTS) of NTU.

\section{References}


1. Geim, A.K., Graphene: status and prospects. Science, 2009. 324(5934): p. 1530-1534.

2. Park, S. and R.S. Ruoff, Chemical methods for the production of graphenes Corrigendum. Nature Nanotechnology, 2010. 5(4): p. 309.

3. McAllister, M.J., et al., Single sheet functionalized graphene by oxidation and thermal expansion of graphite. Chemistry of Materials, 2007. 19(18): p. 4396-4404.

4. Lee, C., X.D. Wei, J.W. Kysar and J. Hone,, Measurement of the elastic properties and intrinsic strength of monolayer graphene. Science, 2008. 321(5887): p. 385-388.

5. Eswaraiah, V., K. Balasubramaniam, and S. Ramaprabhu, One-pot synthesis of conducting graphene-polymer composites and their strain sensing application. Nanoscale, 2012. 4(4): p. 1258-1262.

6. Li, C.X., J. Adamcik, and R. Mezzenga, Biodegradable nanocomposites of amyloid fibrils and graphene with shape-memory and enzyme-sensing properties. Nature Nanotechnology, 2012. 7(7): p. 421-427.

7. Liang, J.J., Y. Wang, Y. Huang, Y. Ma, Z. Liu, J. Cai, C. Zhang, H. Gao and Y. ChenElectromagnetic interference shielding of graphene/epoxy composites. Carbon, 2009. 47(3): p. 922-925.

8. $\quad$ Liang, J.J., Y. Xu , Y. Huang, L. Zhang, Y. Wang, Y. Ma, F. Li, T. Guo and Y. Chen * Infrared-Triggered Actuators from Graphene-Based Nanocomposites. Journal of Physical Chemistry C, 2009. 113(22): p. 9921-9927.

9. Xiao, X.C., T. Xie, and Y.T. Cheng, Self-healable graphene polymer composites. Journal of Materials Chemistry, 2010. 20(17): p. 3508-3514.

10. Zhou, T., X.M. Zhou, and D. Xing, Controlled release of doxorubicin from graphene oxide based charge-reversal nanocarrier. Biomaterials, 2014. 35(13): p. 4185-4194.

11. Dato, A., et al., Substrate-free gas-phase synthesis of graphene sheets. Nano Letters, 2008. 8(7): p. 2012-2016.

12. Reina, A., X. Jia, J. Ho, D. Nezich, H. Son, V. Bulovic, M.S. Dresselhaus and J. Kong, Large area, few-layer graphene films on arbitrary substrates by chemical vapor deposition. Nano Letters, 2009. 9(1): p. 30-35.

13. Novoselov, K.S., A.K. Geim, S.V. Morozov, D.Jiang, Y. Zhang, S.V. Dubonos, I.V. Grigorieva, and A.A. Firsov, Electric field effect in atomically thin carbon films. Science, 2004. 306(5696): p. 666-669.

14. Park, S. and R.S. Ruoff, Chemical methods for the production of graphenes. Nature Nanotechnology, 2009. 4(4): p. 217-224.

15. Erickson, K., R. Erni, Z. Lee, N. Alem, W. Gannett and A. Zettl, Determination of the local chemical structure of graphene oxide and reduced graphene oxide. Advanced Materials, 2010. 22(40): p. 4467-4472.

16. Szabo, T., E. Tombácz, E. Illés and I. Dékány, Enhanced acidity and pH-dependent surface charge characterization of successively oxidized graphite oxides. Carbon, 2006. 44(3): p. 537-545.

17. Titelman, G.I., V. Gelman, S. Bron, R.L. Khalfin, Y. Cohen and H. Bianco-Peled, Characteristics and microstructure of aqueous colloidal dispersions of graphite oxide. Carbon, 2005. 43(3): p. 641-649.

18. Stankovich, S., R.D. Pinera, S.B.T. Nguyen, R.S. Ruoff, Synthesis and exfoliation of isocyanate-treated graphene oxide nanoplatelets. Carbon, 2006. 44(15): p. 3342-3347.

19. Šebenik, U. and M. Krajnc, Influence of the soft segment length and content on the synthesis and properties of isocyanate-terminated urethane prepolymers. International Journal of Adhesion and Adhesives, 2007. 27(7): p. 527-535.

20. Loh, K.P., Q. Bao, P. Kailian Ang and J. Yang, The chemistry of graphene. Journal of Materials Chemistry, 2010. 20(12): p. 2277-2289. 
21. Dreyer, D.R., S. Park, C.W. Bielawski and R.S. Ruoff, The chemistry of graphene oxide. Chemical Society Reviews, 2010. 39(1): p. 228-240.

22. Jing, Q., J.Y. Law, L.P. Tan, V.V. Silberschmidt, L. Li and Z.L. Dong Preparation, characterization and properties of polycaprolactone diol-functionalized multi-walled carbon nanotube/thermoplastic polyurethane composite. Composites Part A: Applied Science and Manufacturing, 2015. 70(0): p. 8-15.

23. Pan, Y.Z., H. Bao, N.G. Sahoo, T. Wu and L Li, Water-soluble poly(Nisopropylacrylamide)-graphene sheets synthesized via click chemistry for drug delivery. Advanced Functional Materials, 2011. 21(14): p. 2754-2763.

24. Salavagione, H.J., M.A. Gomez, and G. Martinez, Polymeric modification of graphene through esterification of graphite oxide and poly(vinyl alcohol). Macromolecules, 2009. 42(17): p. 6331-6334.

25. Liu, L.H., M.M. Lerner, and M.D. Yan, Derivitization of Pristine Graphene with Well-Defined Chemical Functionalities. Nano Letters, 2010. 10(9): p. 3754-3756.

26. Jung, I., D. Dikin, S. Park, W. Cai, S.L. Mielke and R.S. Ruoff Effect of ater vapor on electrical properties of individual reduced graphene oxide sheets. Journal of Physical Chemistry C, 2008. 112(51): p. 20264-20268.

27. Cho, J.W., J.W. Kim, Y.C. Jung and N.S. Goo, Electroactive shape-memory polyurethane composites incorporating carbon nanotubes. Macromolecular Rapid Communications, 2005. 26(5): p. 412-416.

28. Blagbrough, I.S., N.E. Mackenzie, C. Ortiz and A.I. Scott, The condensation reaction between isocyanates and carboxylic-acids - a practical synthesis of substituted amides and anilides. Tetrahedron Letters, 1986. 27(11): p. 1251-1254.

29. March, J. and M.B. Smith, March's advanced organic chemistry : reactions, mechanisms and structure. 2007, Hoboken, N.J: Wiley.

30. Silverstein, R.M., F.X. Webster, and D.J. Kiemle, Spectrometric identification of organic compounds [electronic resource] / R.M. Silverstein, F.X. Webster, D.J. Kiemle. 2005: Hoboken, NJ : John Wiley \& Sons, c2005.7th ed.

31. Yun, S., H. Im, and J. Kim, The effect of different hard segments in polyurethane on the electrical conductivity of polyurethane grafted multi-walled carbon nanotube/polyurethane nanocomposites. Synthetic Metals, 2011. 161(13-14): p. 13611367.

32. Yang, L.P., W.A. Yee, S.L. Phua, J. Kong, H. Ding, J.W. Cheah and X. Lu, A high throughput method for preparation of highly conductive functionalized graphene and conductive polymer nanocomposites. Rsc Advances, 2012. 2(6): p. 2208-2210.

33. Wu, C., X. Huang, G. Wang, X. Wu, K. Yang, S. Li and P. Jiang Hyperbranchedpolymer functionalization of graphene sheets for enhanced mechanical and dielectric properties of polyurethane composites. Journal of Materials Chemistry, 2012. 22(14): p. 7010-7019.

34. Zhou, D., Q.-Y. Cheng, and B.-H. Han, Solvothermal synthesis of homogeneous graphene dispersion with high concentration. Carbon, 2011. 49(12): p. 3920-3927.

35. Pei, S. and H.-M. Cheng, The reduction of graphene oxide. Carbon, 2012. 50(9): p. 3210-3228.

36. Sahoo, N.G., H.K.F. Cheng, L. Li, S.H. Chan, Z. Judeh and J. Zhao, Specific functionalization of carbon nanotubes for advanced polymer nanocomposites. Advanced Functional Materials, 2009. 19(24): p. 3962-3971.

37. Cao, Y., J. Feng, and P. Wu, Preparation of organically dispersible graphene nanosheet powders through a lyophilization method and their poly(lactic acid) composites. Carbon, 2010. 48(13): p. 3834-3839. 


\section{Figures}

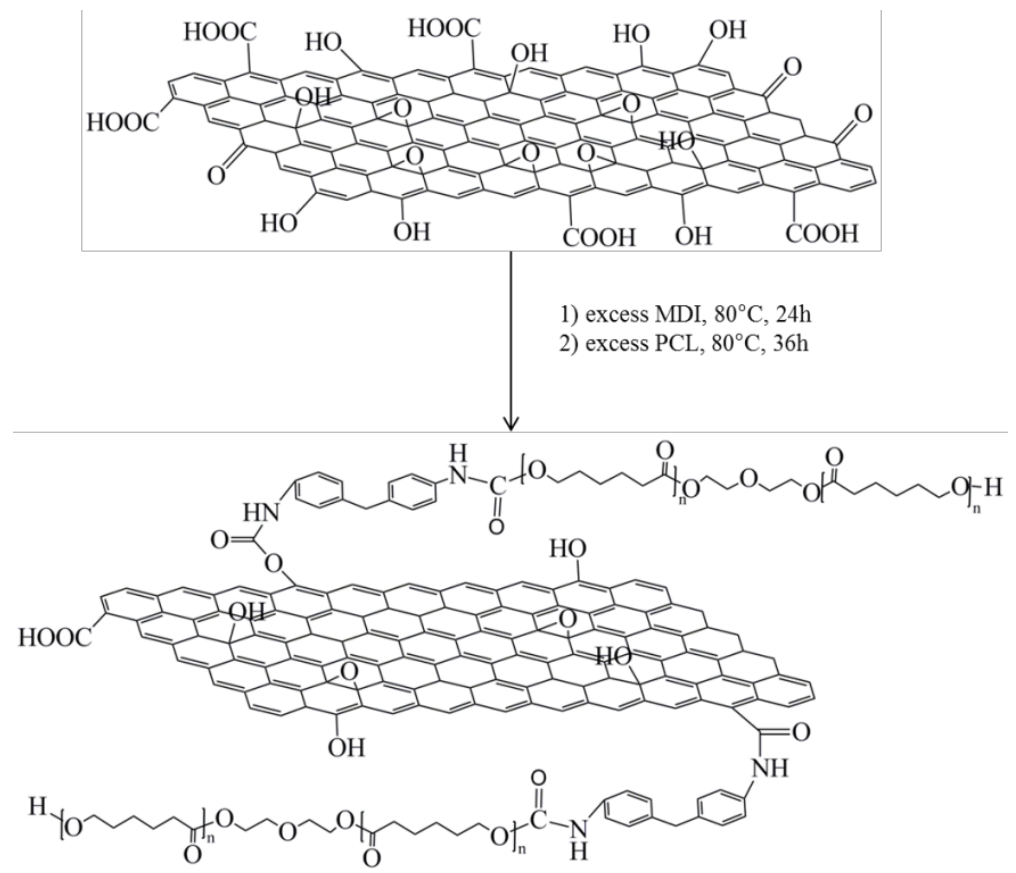

Fig. 1. Schematic of preparing FGO

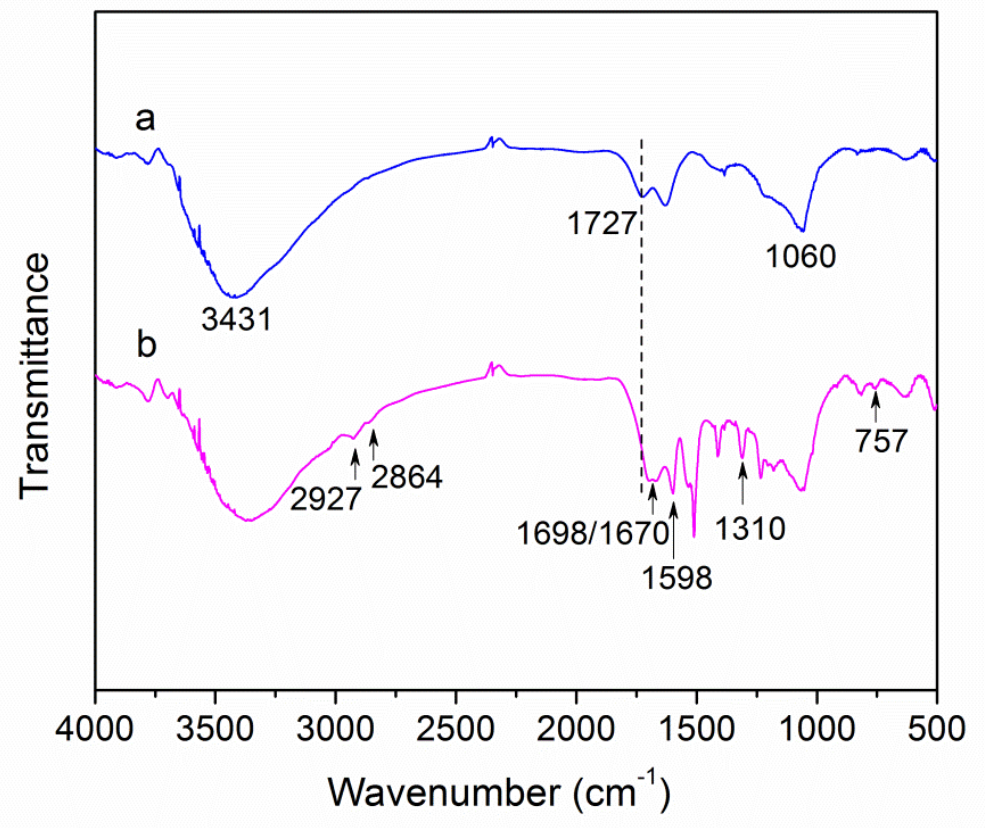

Fig. 2. FTIR spectra of GO (a) and FGO (b) 


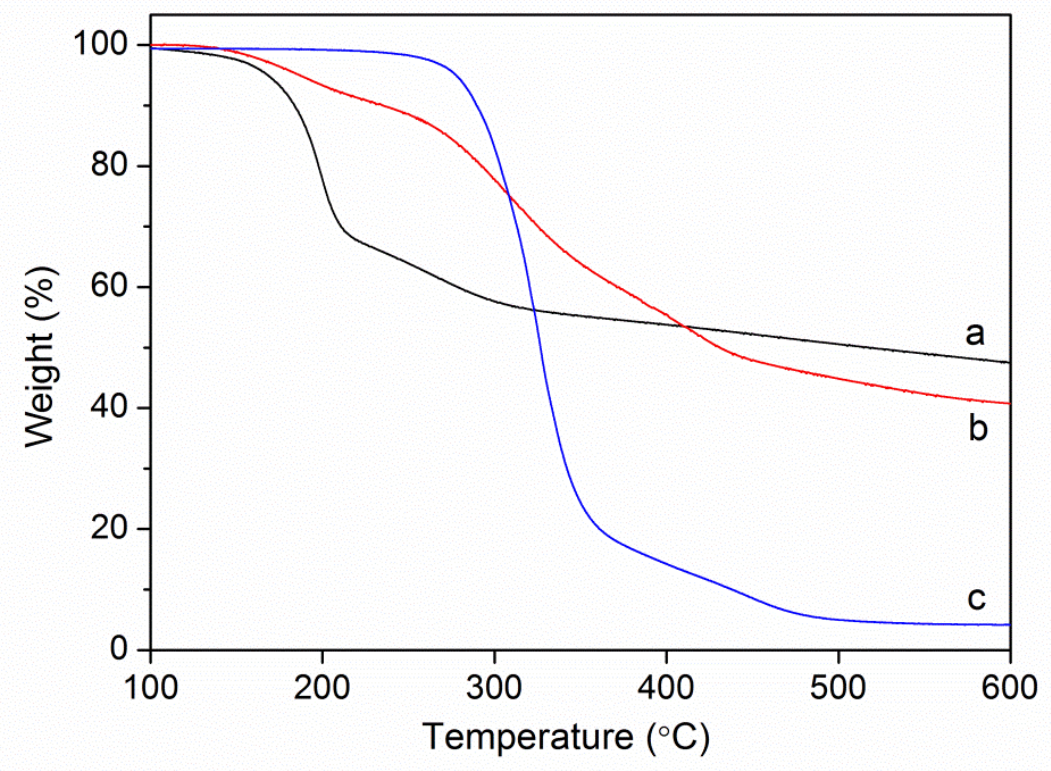

Fig. 3. TGA curves of GO (a), FGO (b) and PU (c)

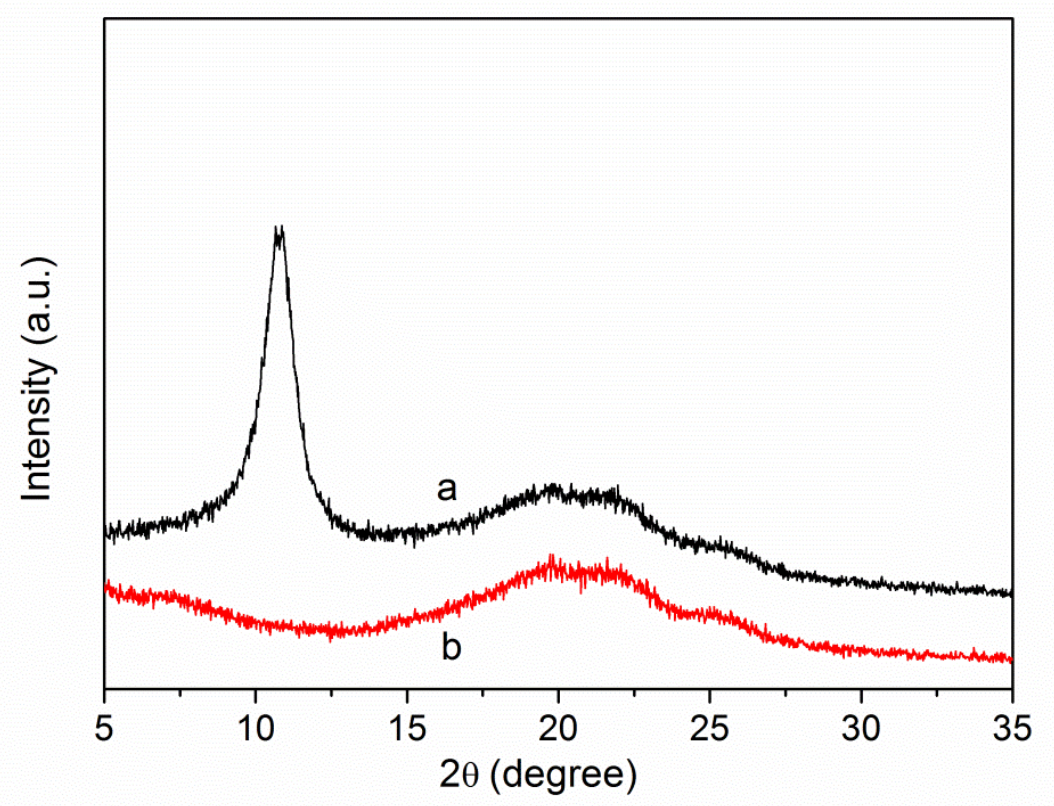

Fig. 4. XRD patterns of GO (a) and FGO (b) 


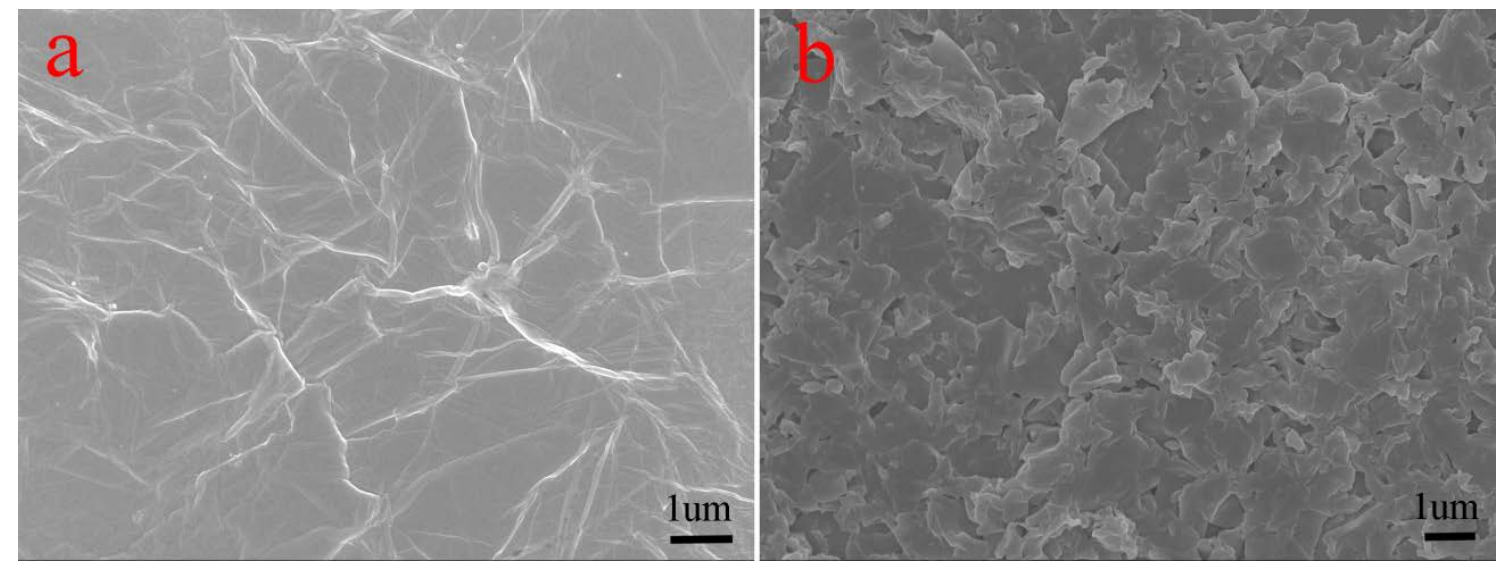

Fig. 5. FESEM images of GO (a) and FGO (b)

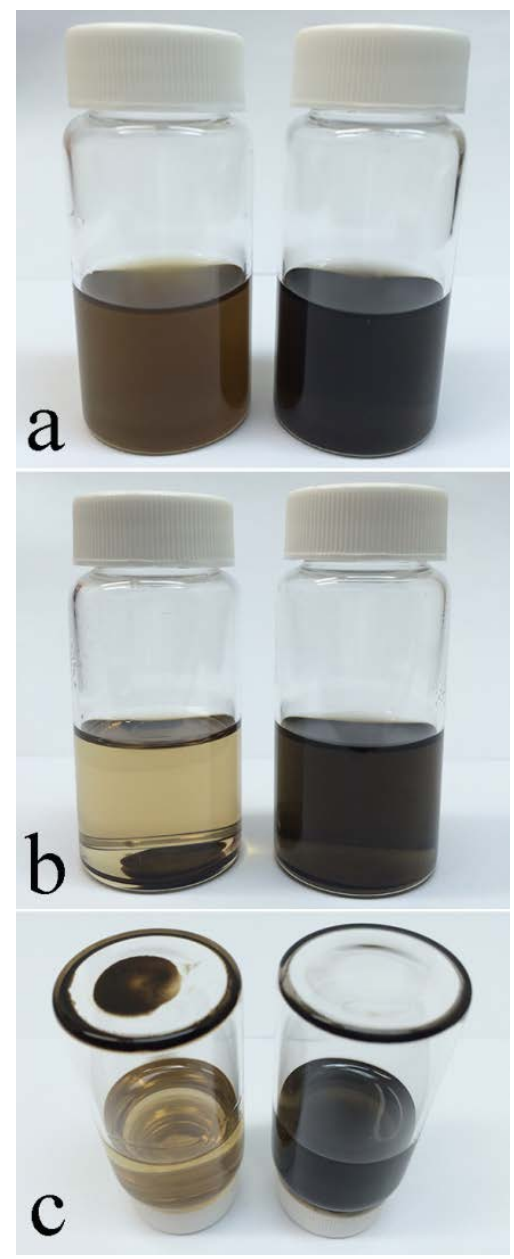

Fig. 6. Digital photographs of GO (left) and FGO (right) dispersed in DMF (0.5 mg/ml): 5 min (a) and 14 days (b, c) after ultrasonication 

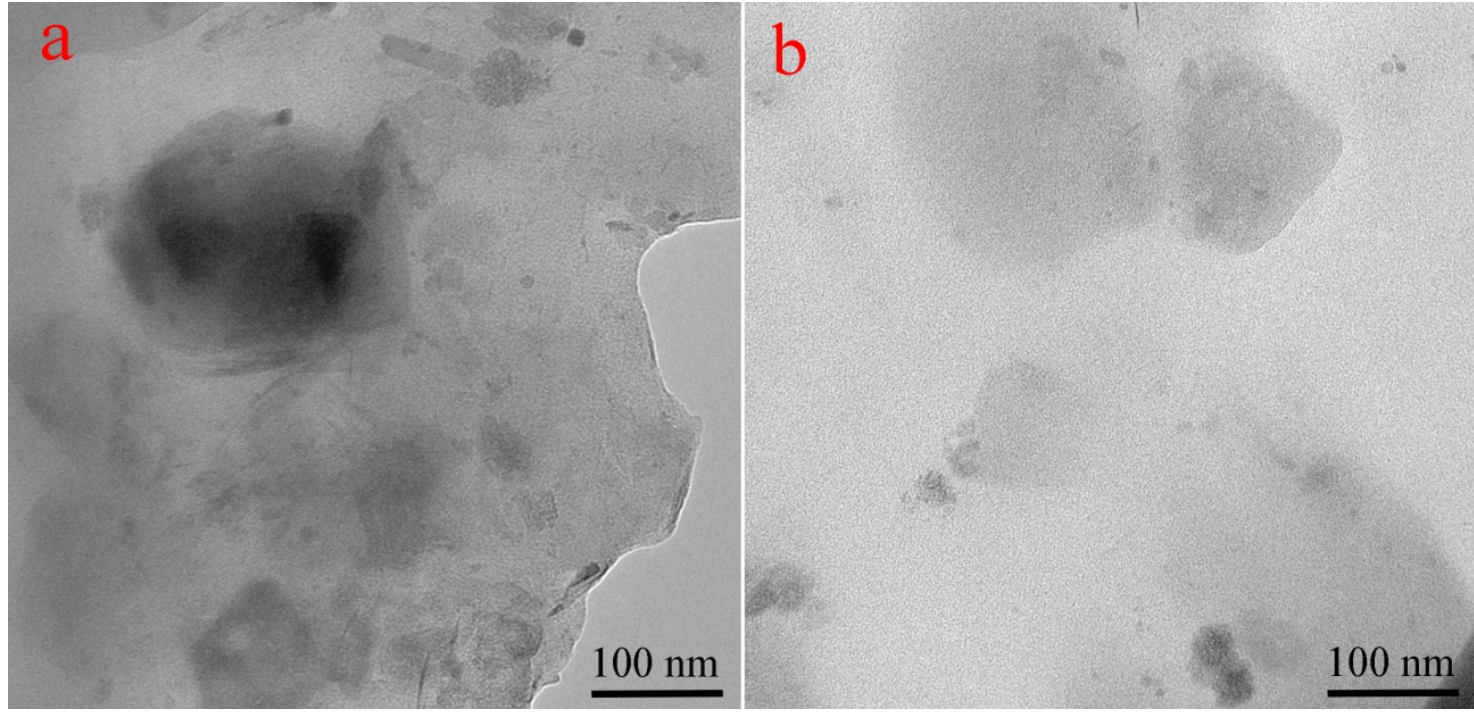

Fig. 7. TEM images of GO/PU 0.4 wt\% composite (a) and FGO/PU 0.4 wt $\%$ composite (b)
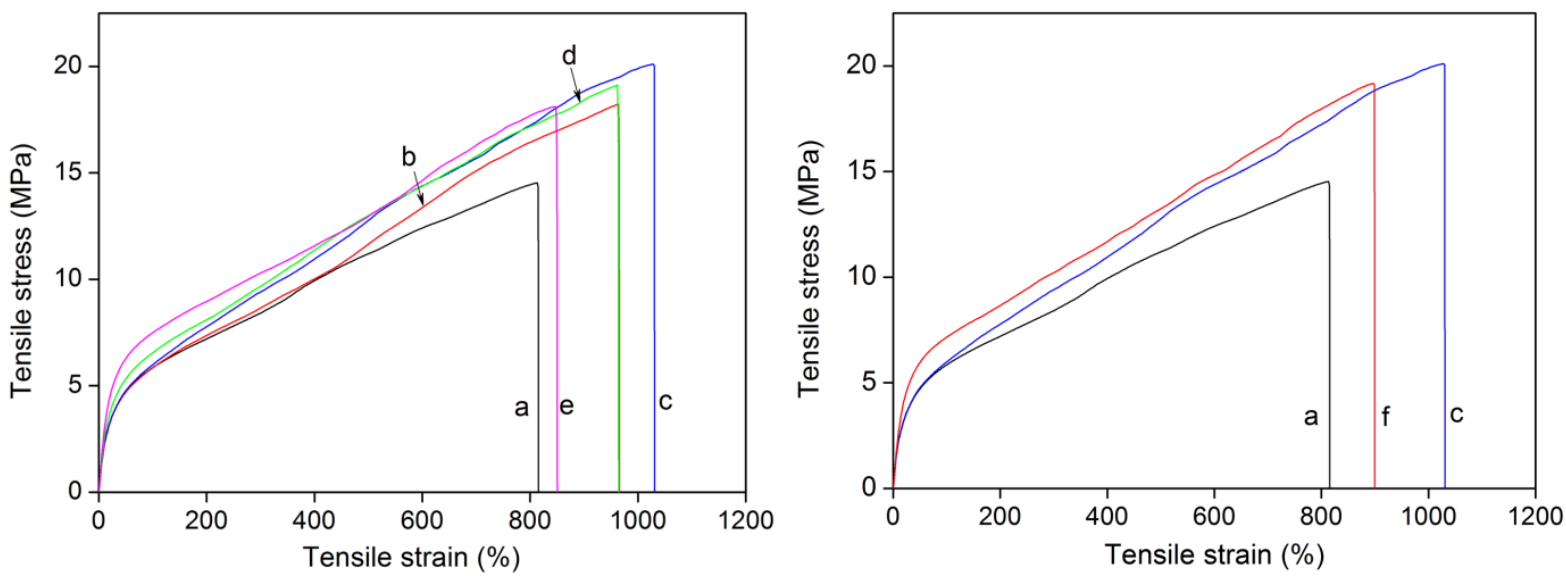

Fig. 8. Typical stress-strain curves: (a) PU; (b)-(e) FGO/PU with different fractions of nanofillers: (b) 0.1 wt\%, (c) 0.4 wt\%, (d) 0.7 wt $\%, 1$ wt $\%$; (f) GO/PU 0.4 wt\% 


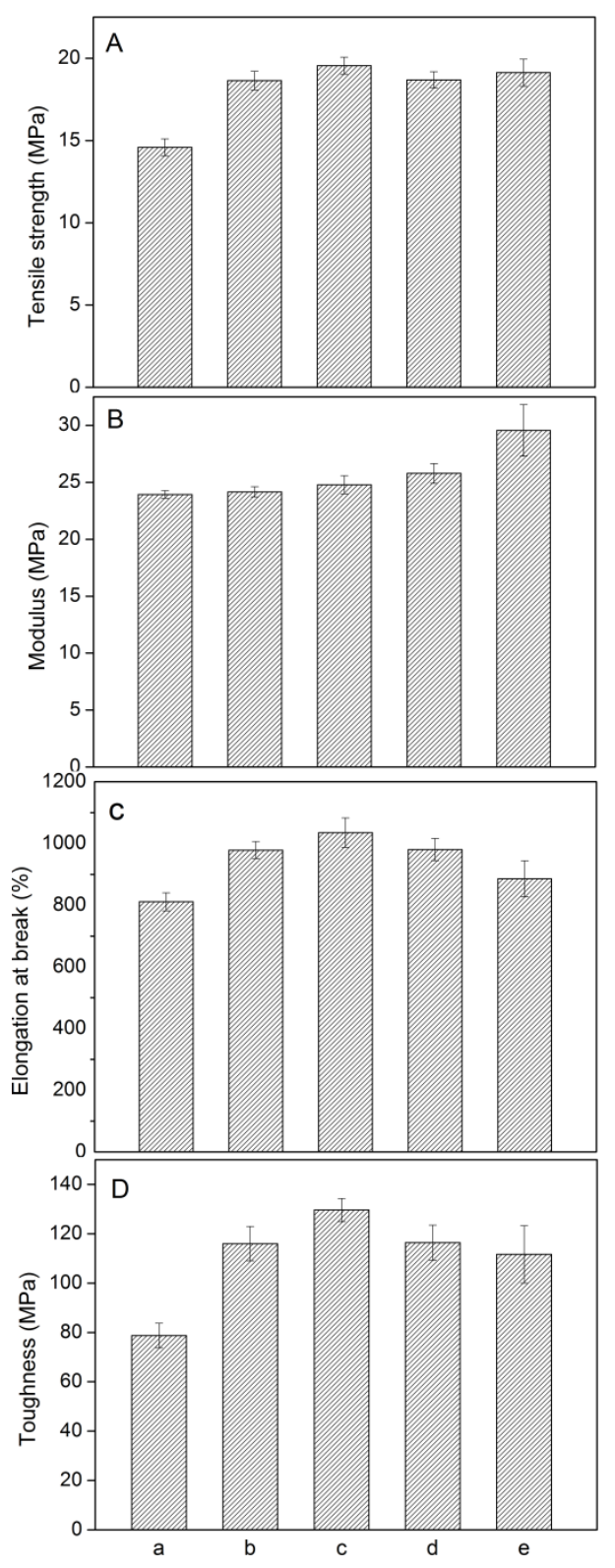

Fig. 9. Tensile strength (A), modulus (B), elongation at break (C) and toughness (D) for studied materials: (a) PU; (b) FGO/PU 0.1 wt\%; (c) FGO/PU 0.4 wt\%; (d) FGO/PU 0.7 wt\%; (e) FGO/PU 1 wt $\%$ 


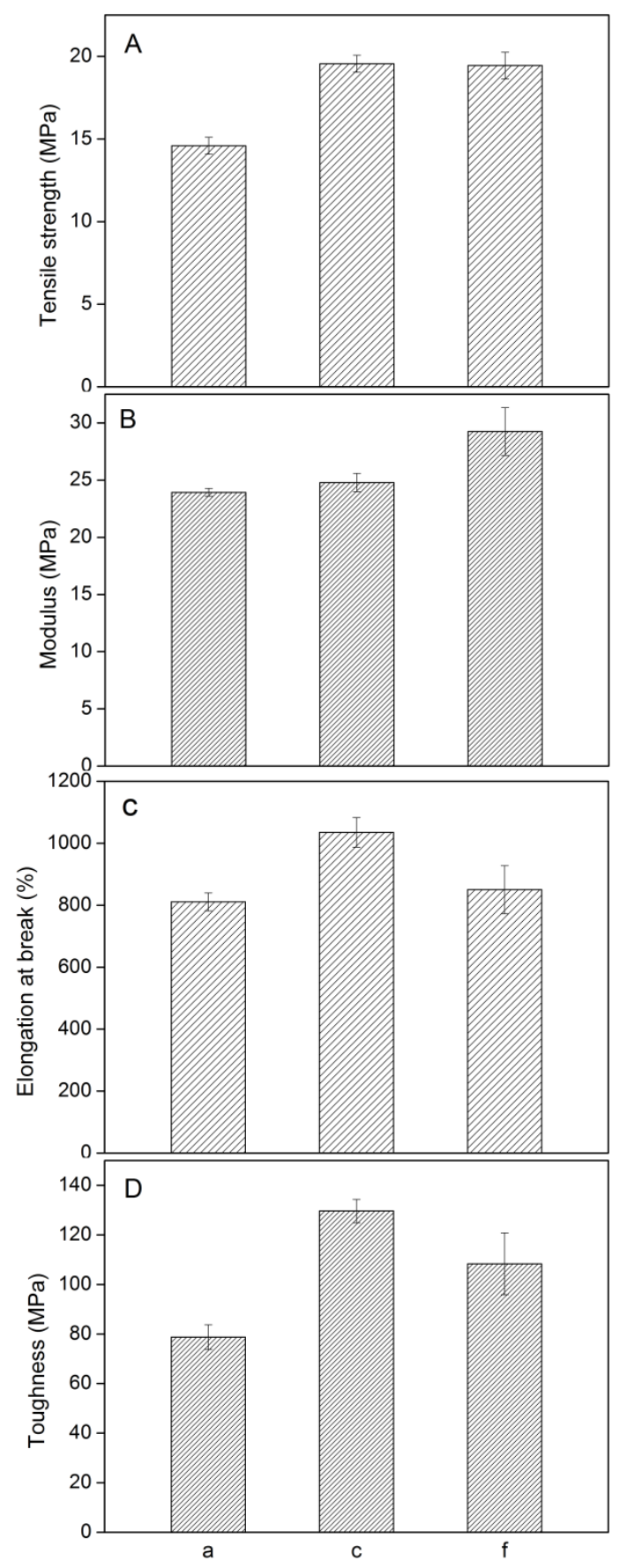

Fig. 10. Tensile strength (A), modulus (B), elongation at break (C) and toughness (D) for studied materials: (a) PU; (c) FGO/PU 0.4 wt\%; (f) GO/PU 0.4 wt\% 


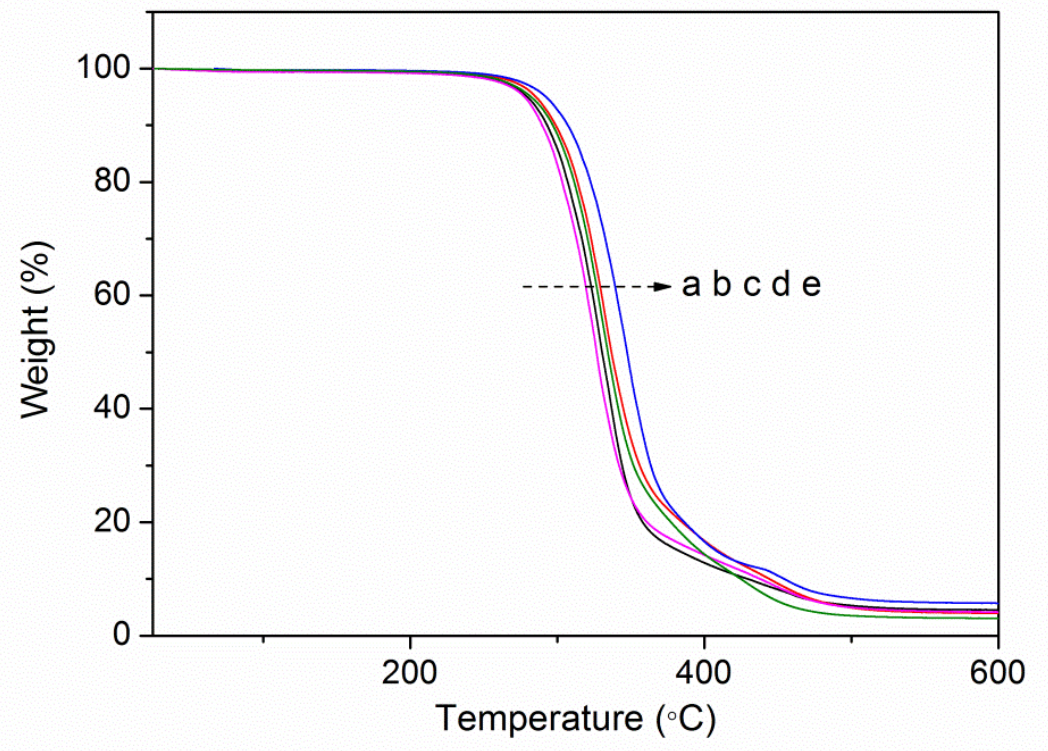

Fig. 11. TGA curves: (a) PU; (b) FGO/PU 0.1 wt\%; (c) FGO/PU 0.4 wt\%; (d) FGO/PU 0.7 wt\%; (e) FGO/PU 1 wt\% 